UDC 355/359

SCOPUS CODE 2205

https://doi.org/10.36073/1512-0996-2020-2-86-96

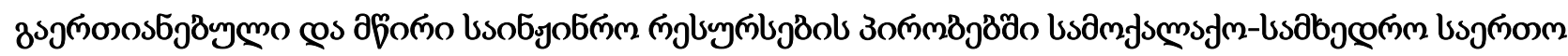

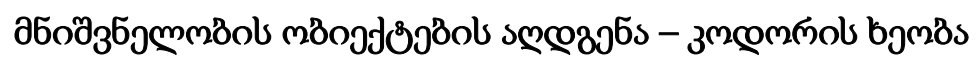

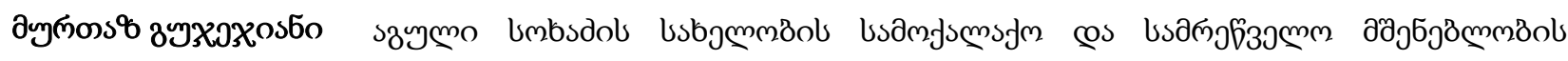

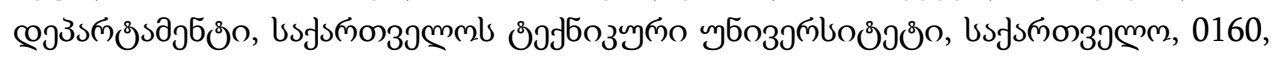

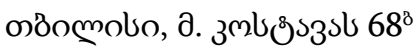

E-mail: nav.qutaisi@gmail.com

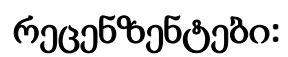

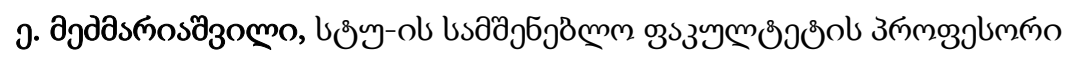

E-mail: e.medzmariashvili@gtu.ge

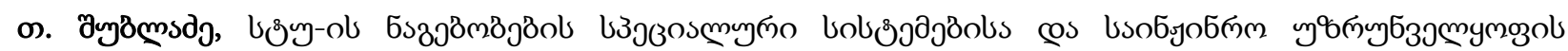

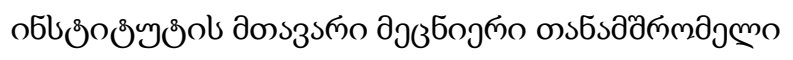

E-mail: shubladze.tengiz@gmail.com

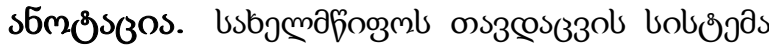

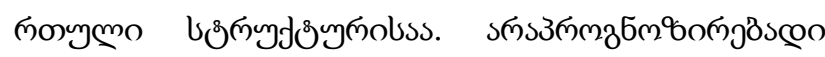

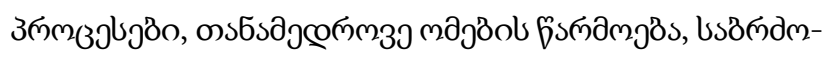

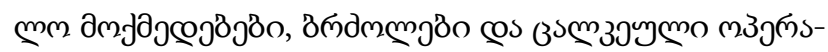

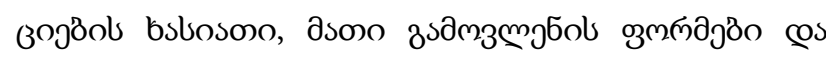

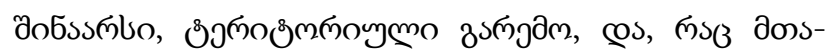

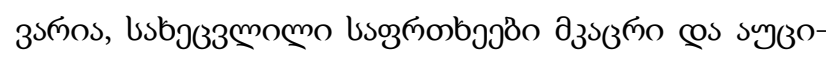

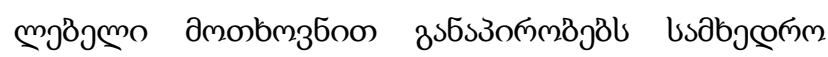

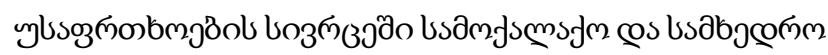

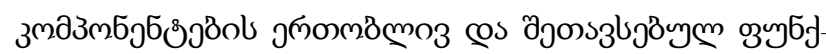

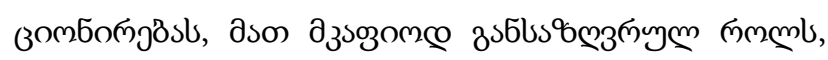

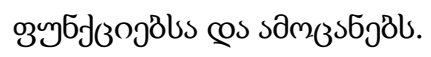

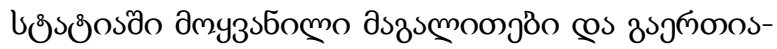

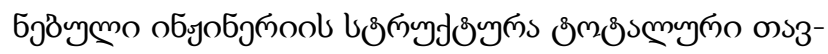

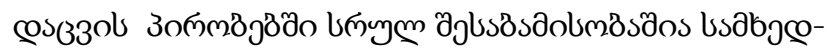

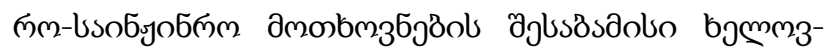

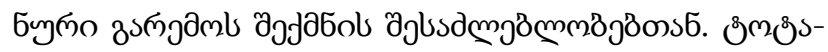

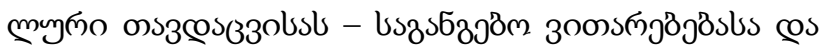

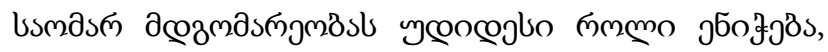

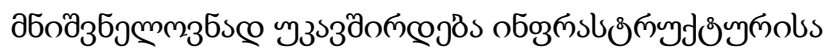

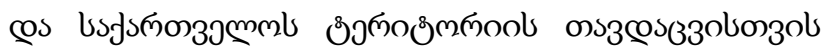

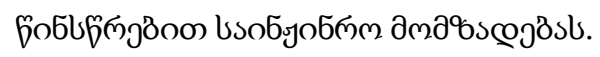

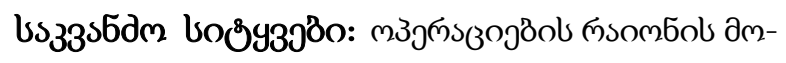

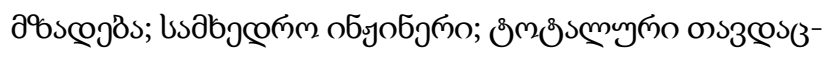

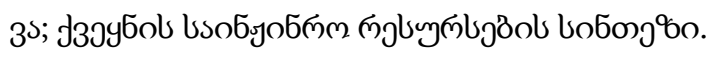




\section{ğbsзsме}

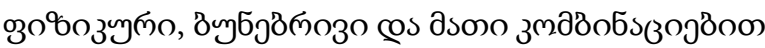

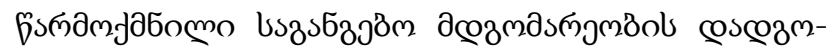

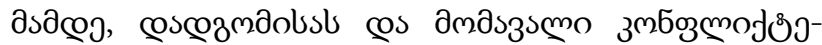

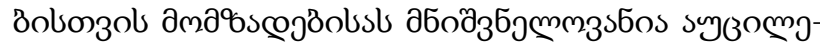

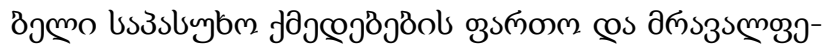

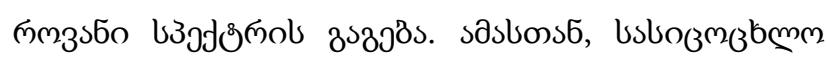

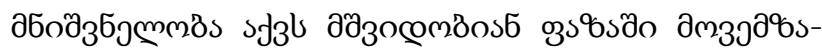

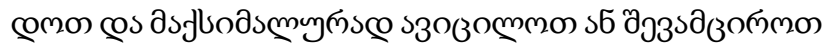

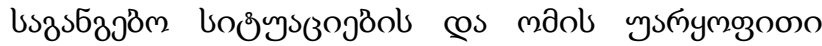

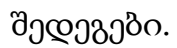

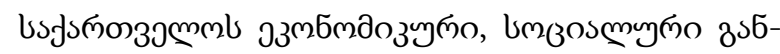

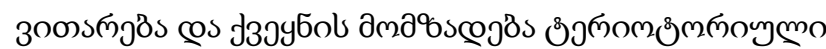

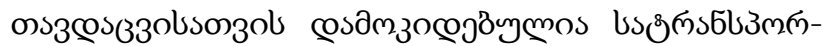

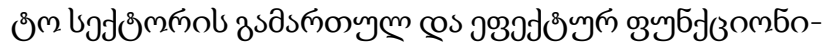

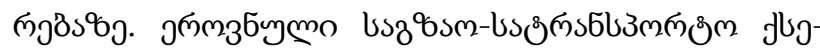

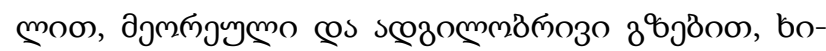

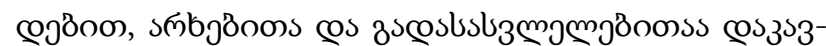

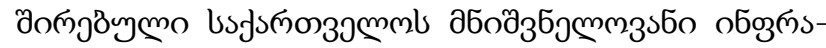

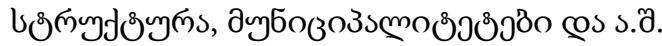

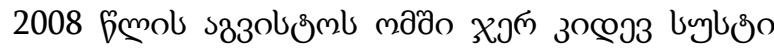

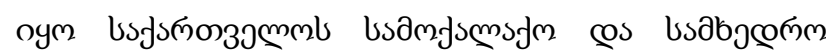

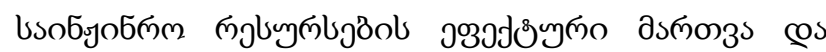

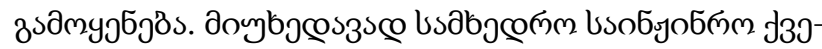

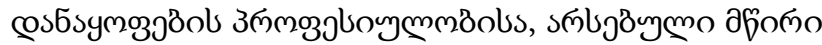

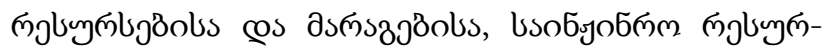

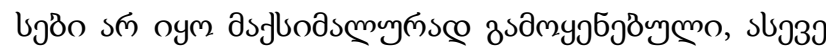

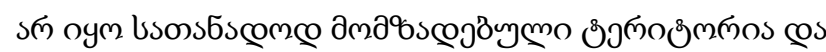

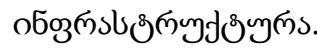

\section{dornooscen 6sfomo}

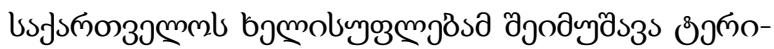

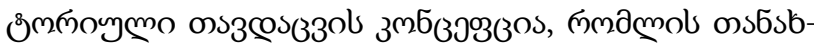

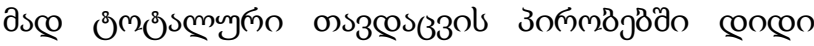

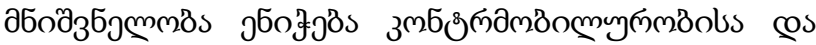

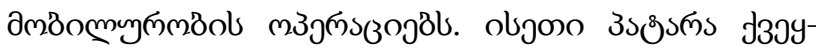

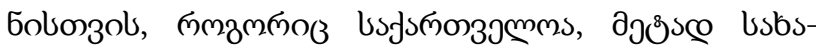

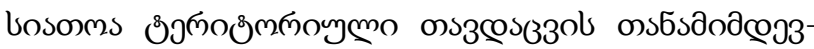

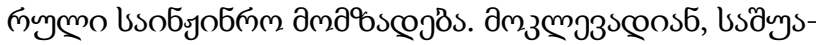

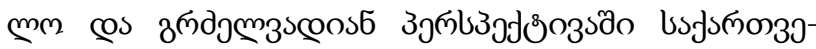

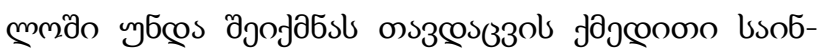

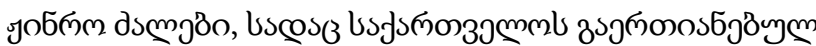

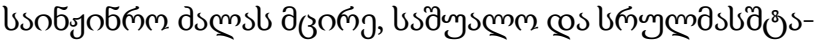

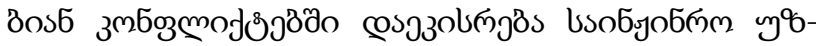

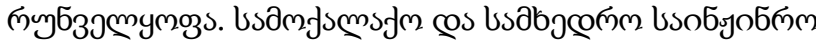

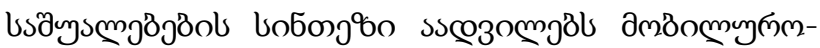

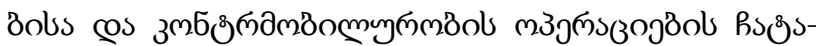

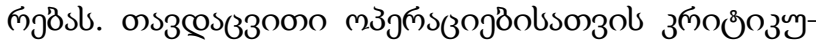

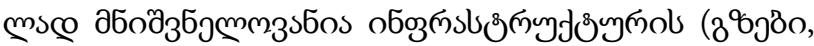

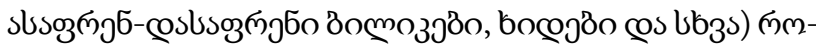

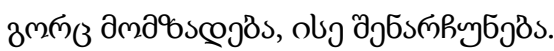

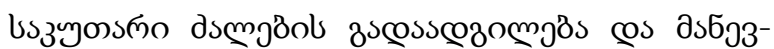

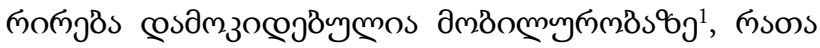

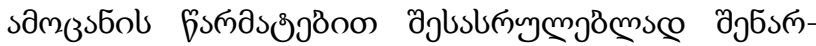

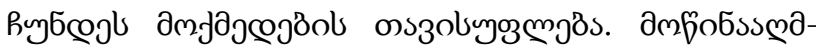

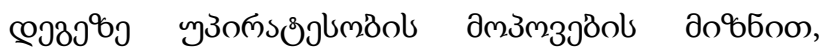

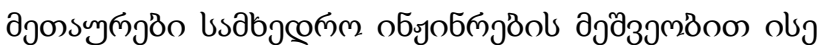

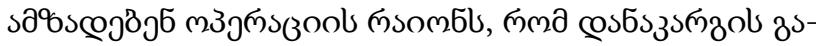

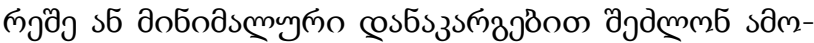

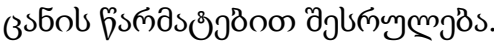

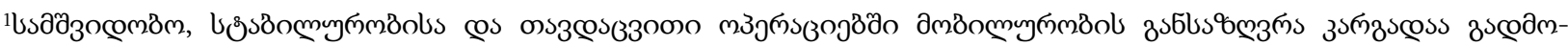

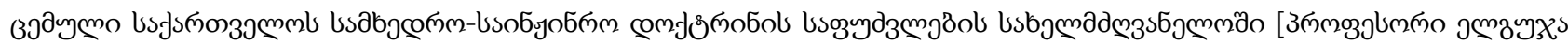

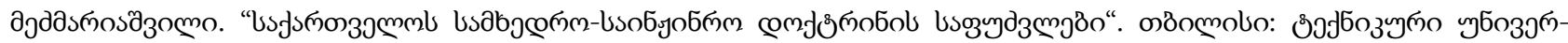

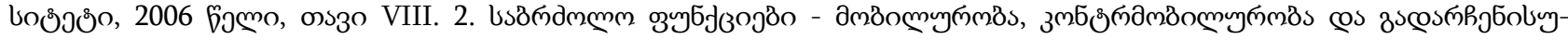

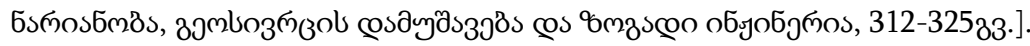




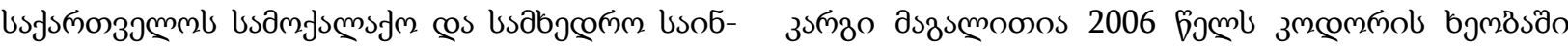

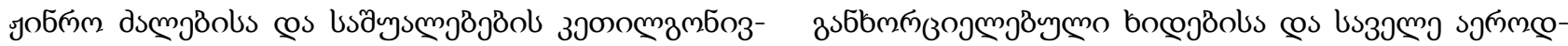

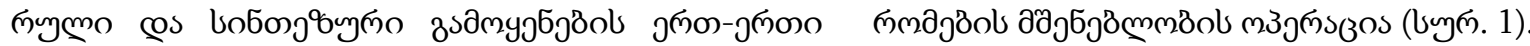

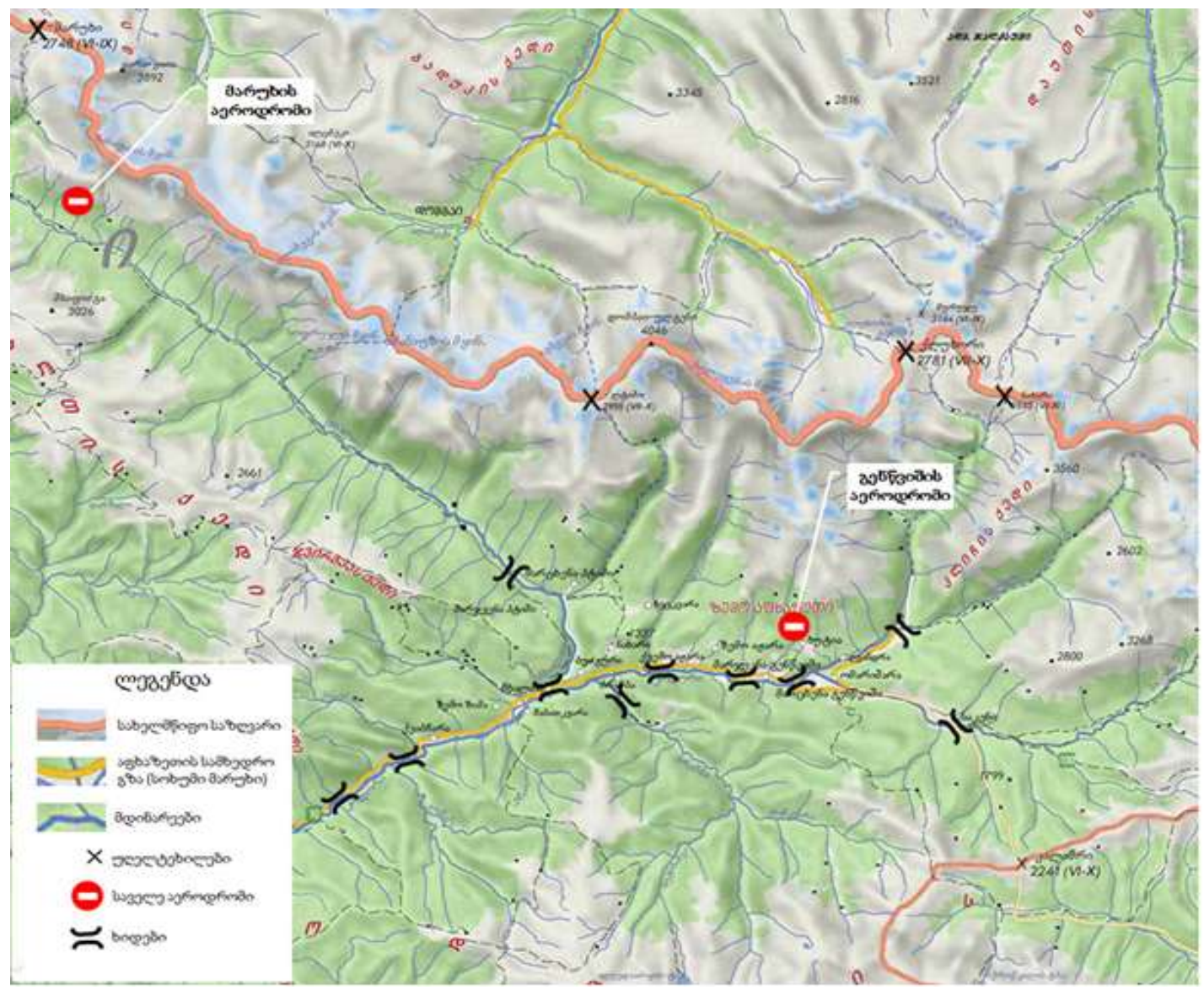

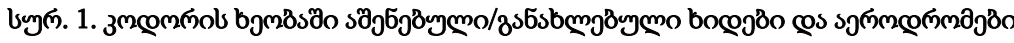

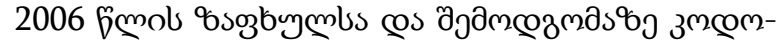

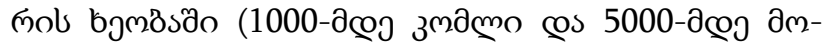

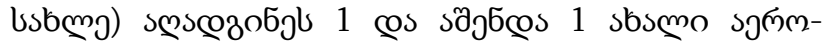

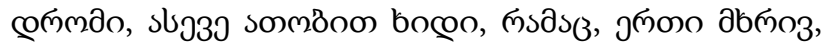

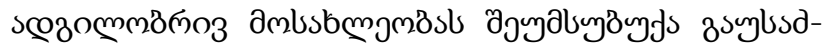

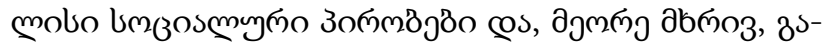

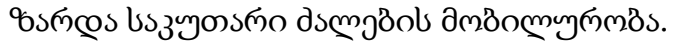

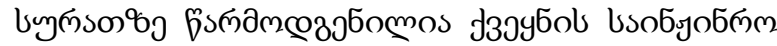

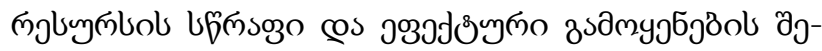

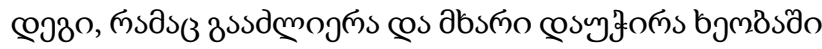

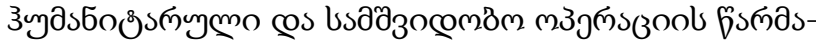

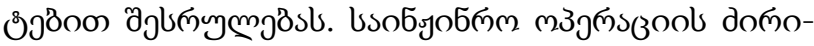

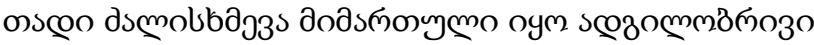

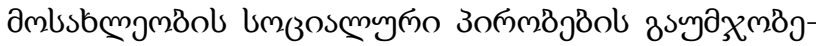

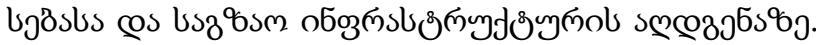

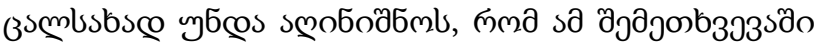

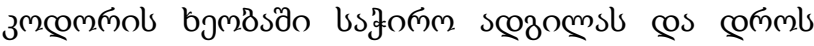

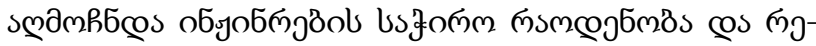

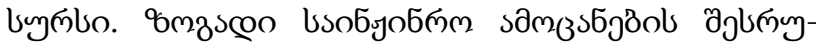

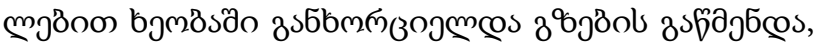




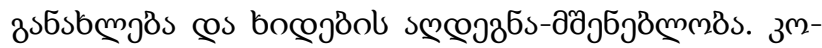

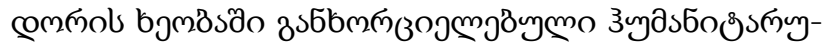

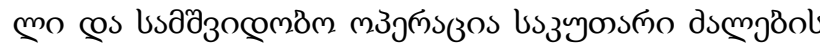

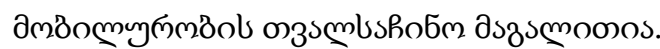

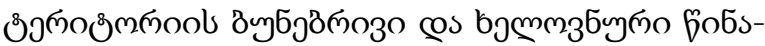

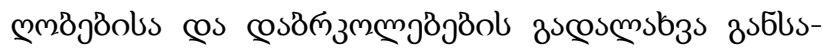

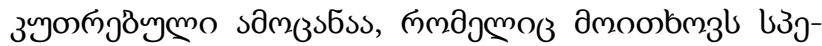

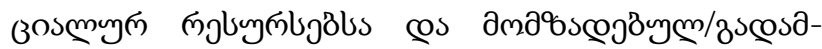

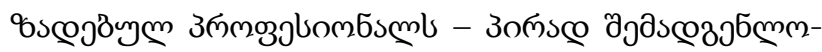

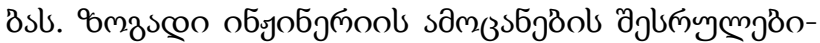

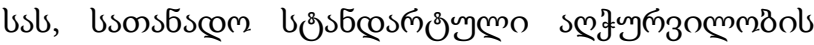

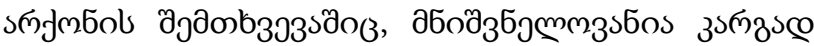

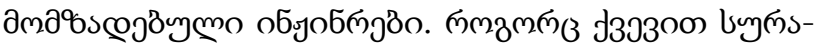

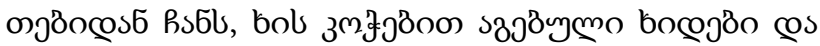

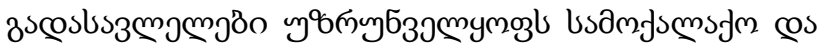

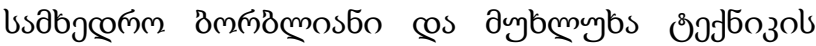

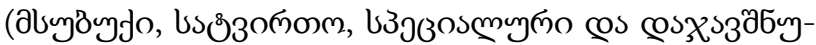

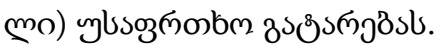

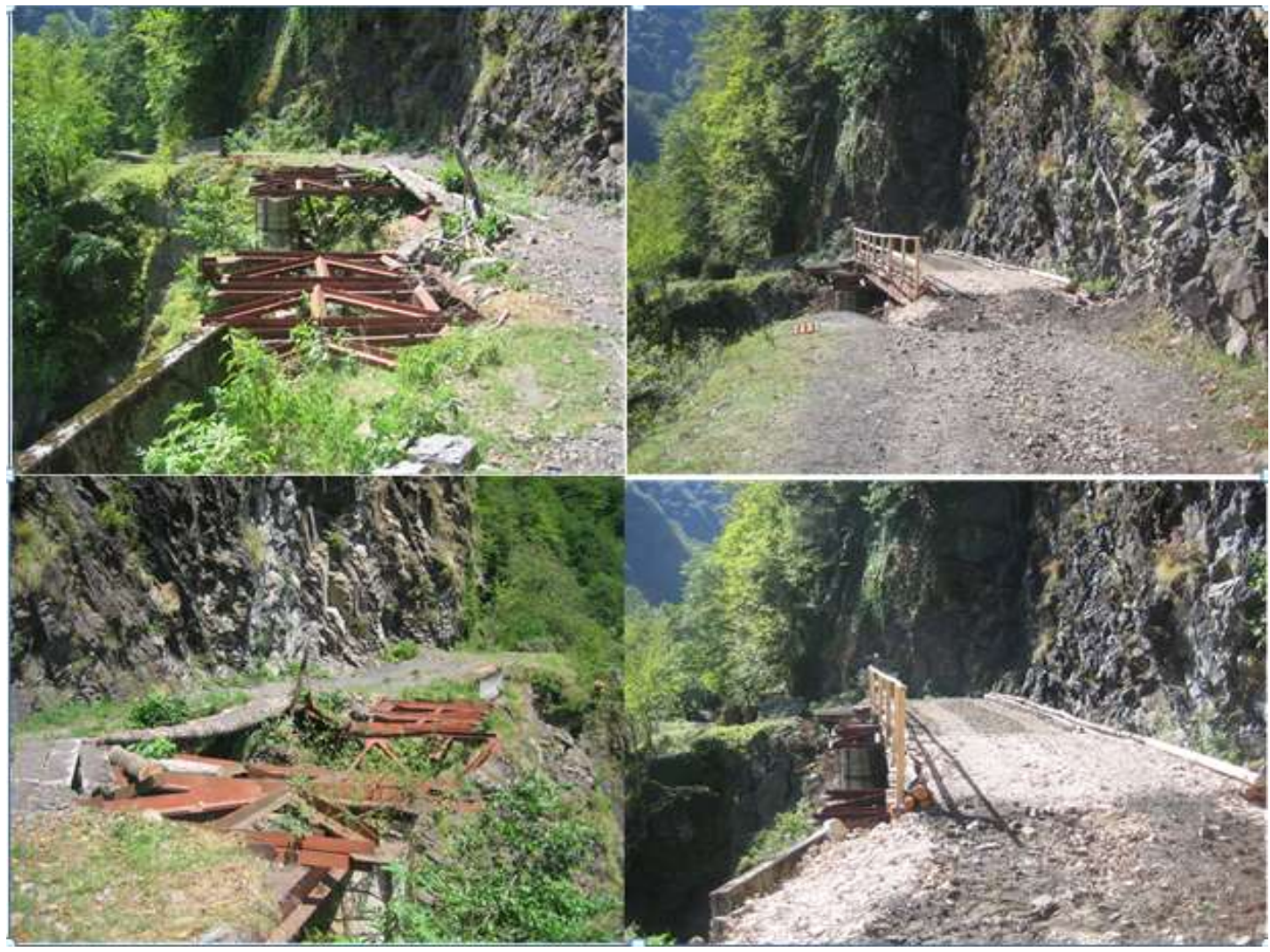

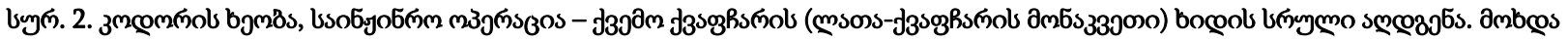

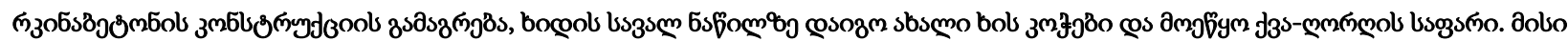

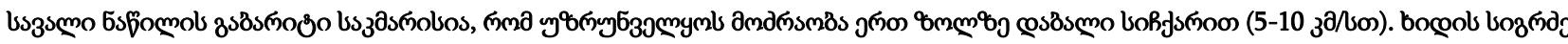

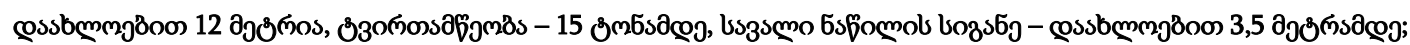

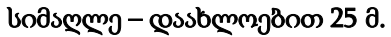




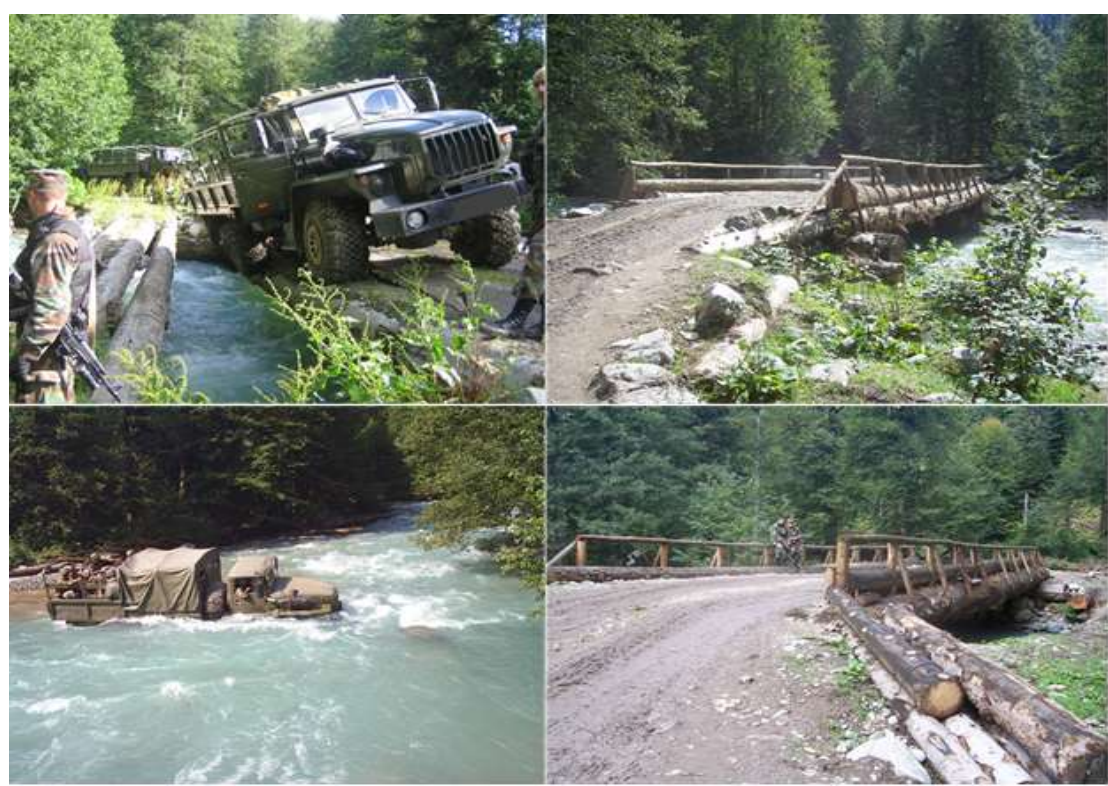

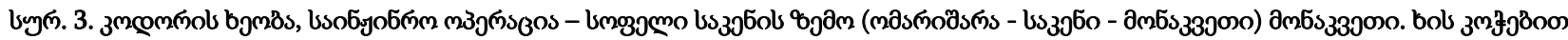

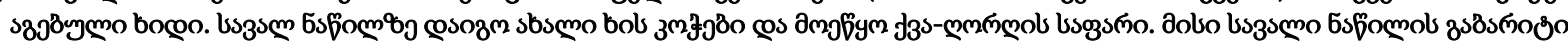

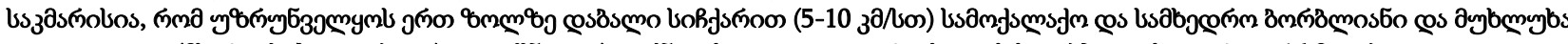

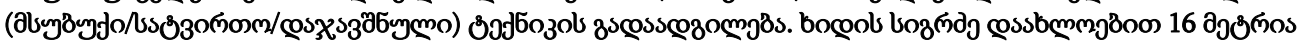

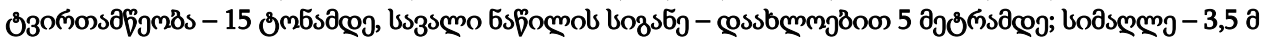

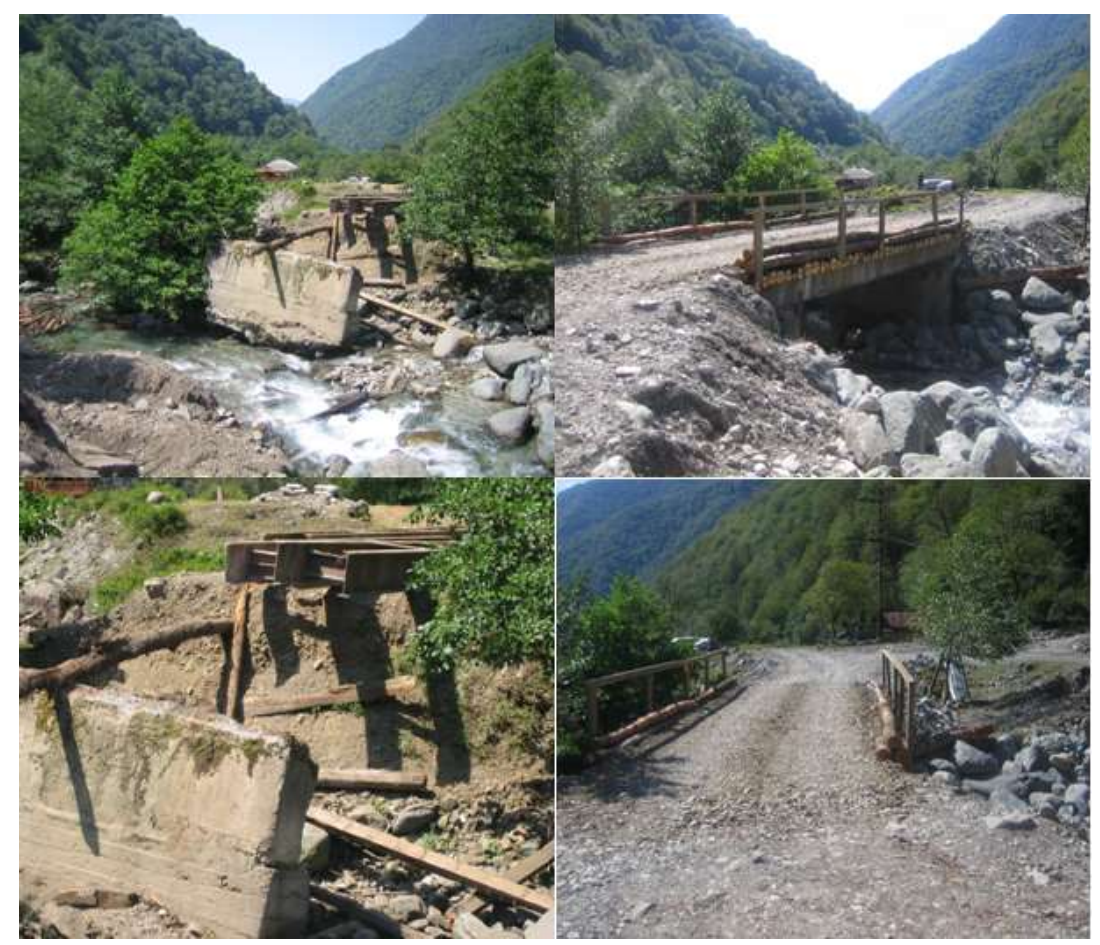

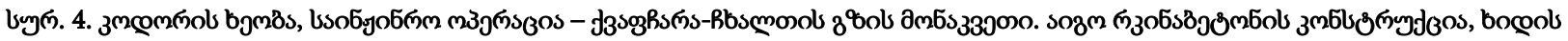

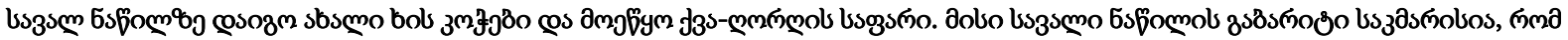

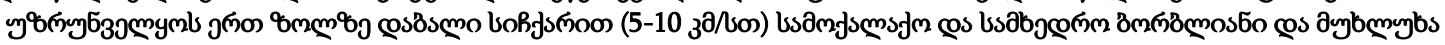

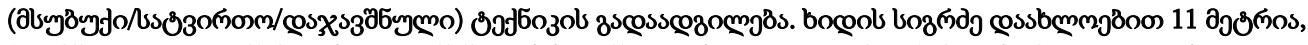

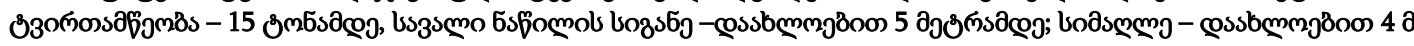




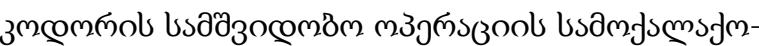

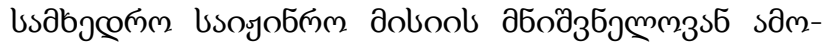

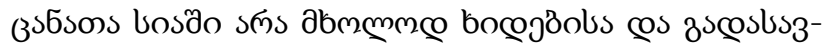

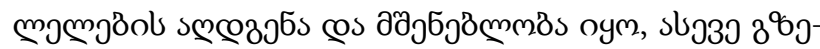

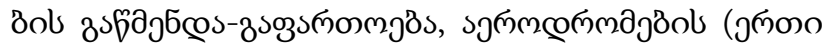

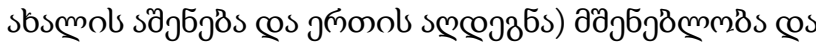

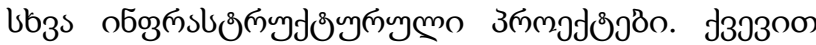

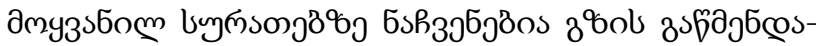

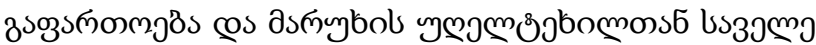

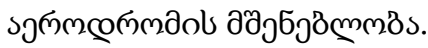

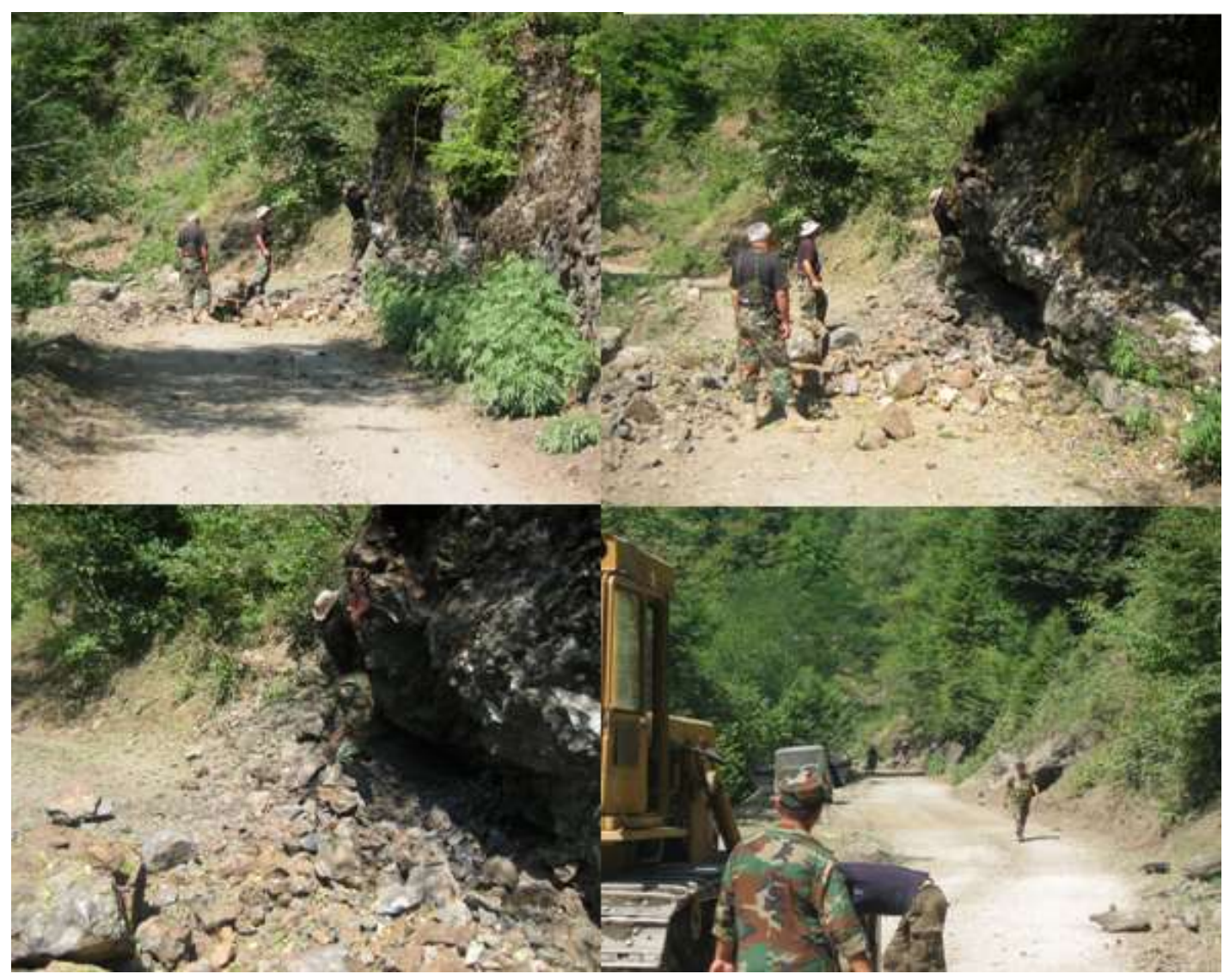

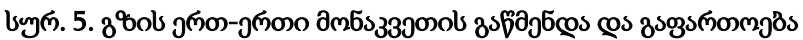

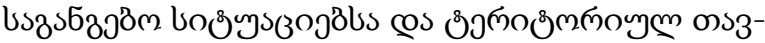

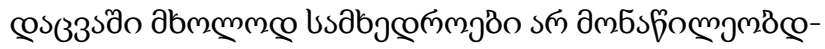

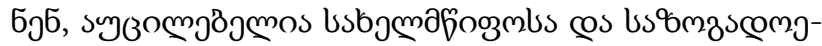

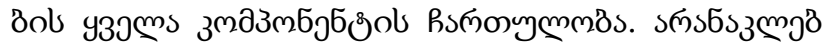

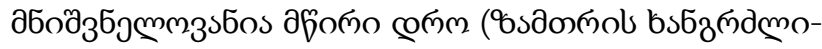

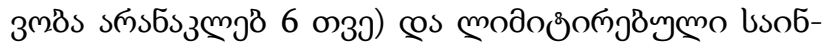

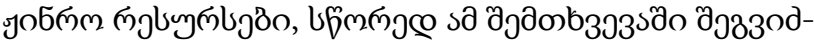

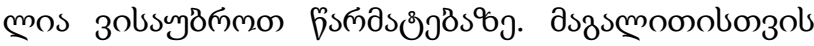

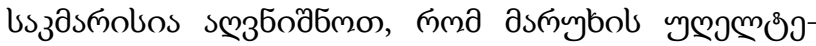

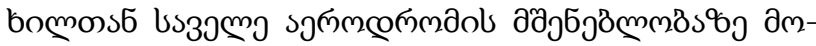

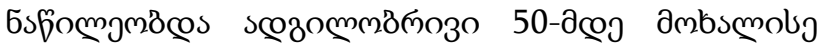

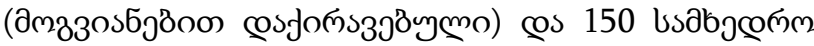

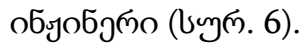




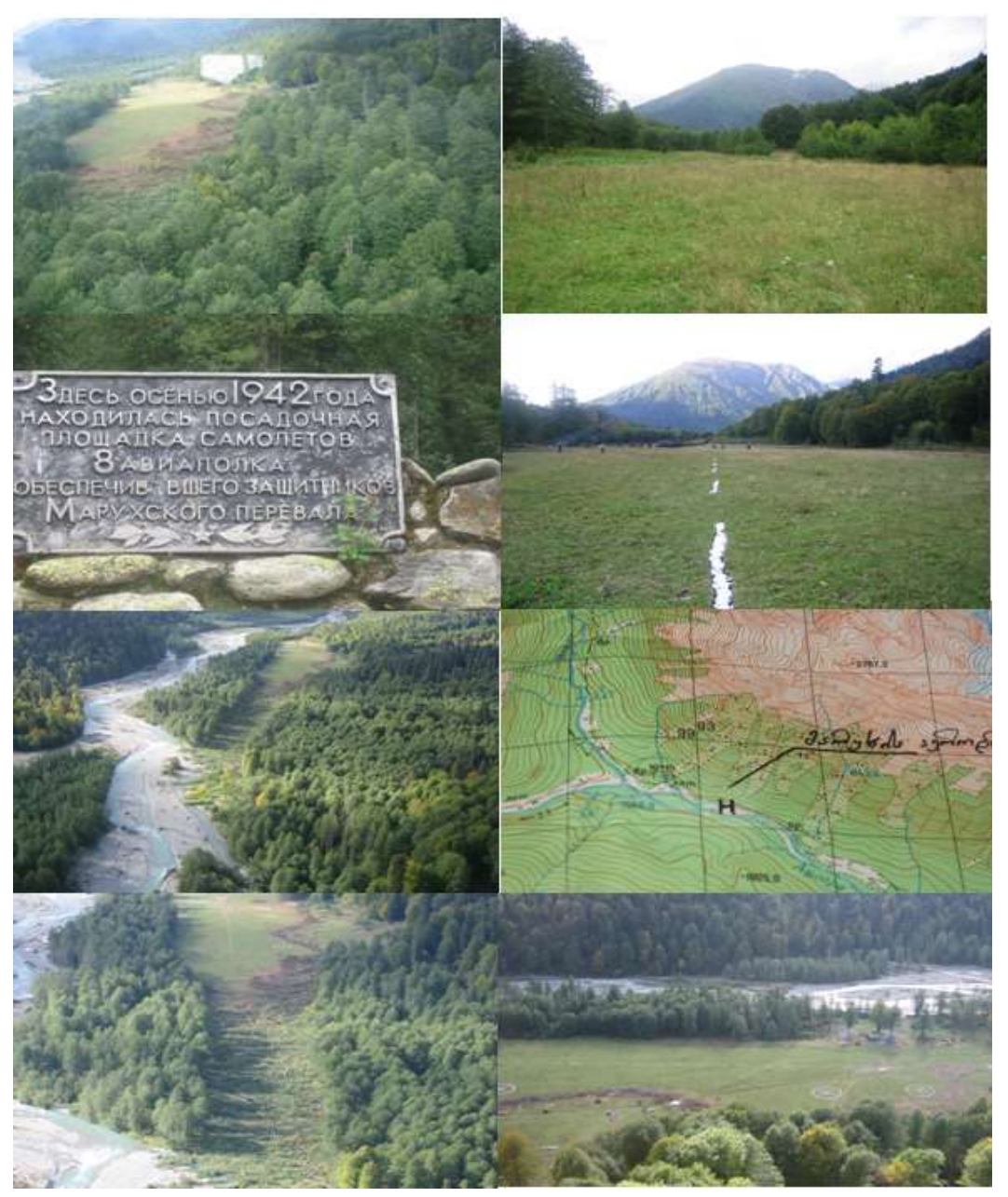

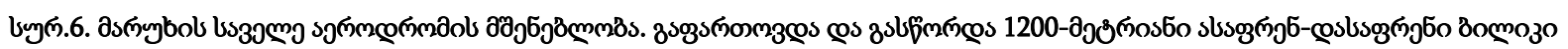

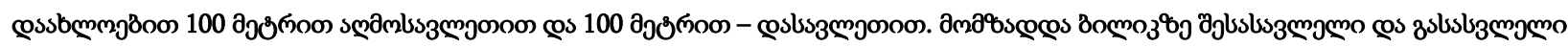

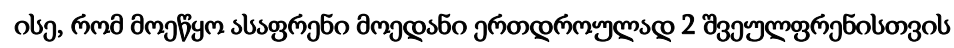

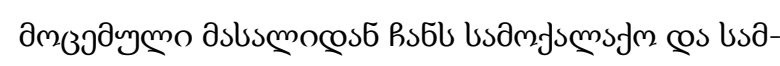

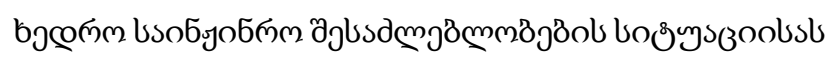

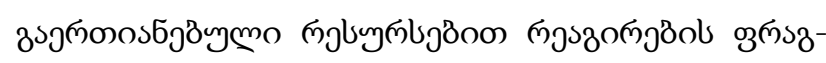

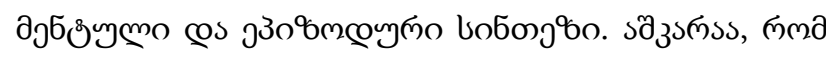

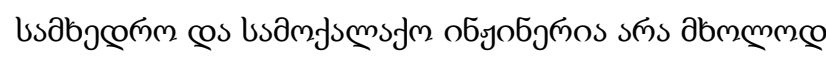

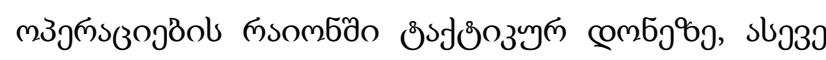

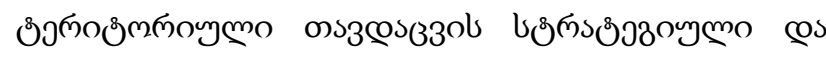

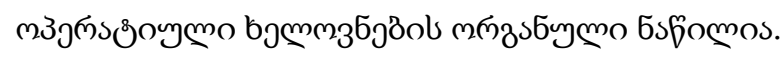

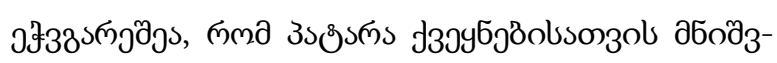

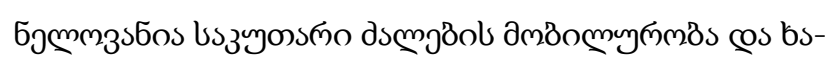

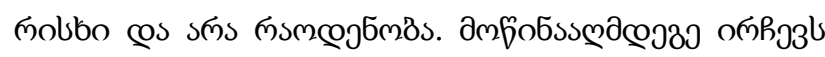

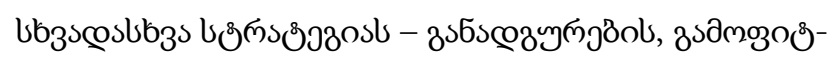

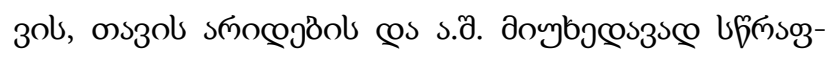

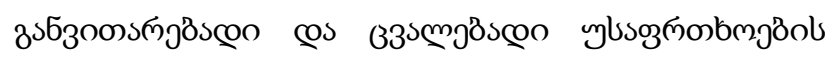

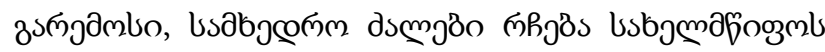

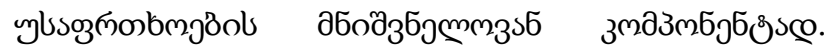




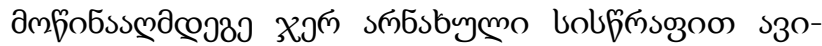
плпృ

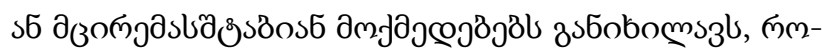

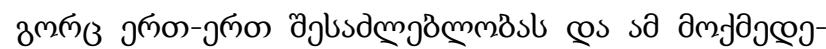

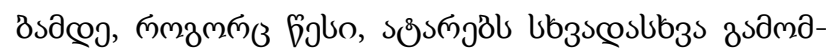

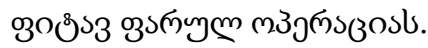

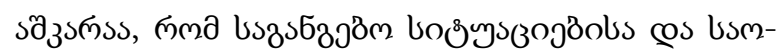

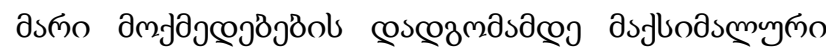

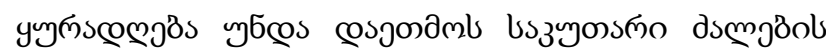

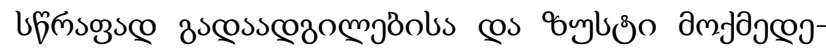

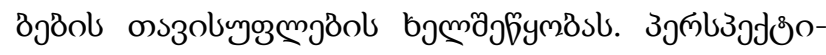

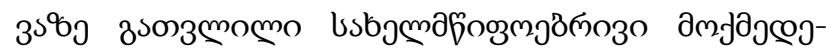

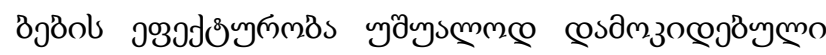

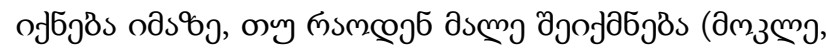

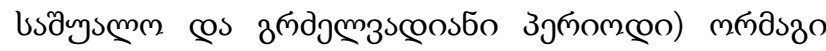

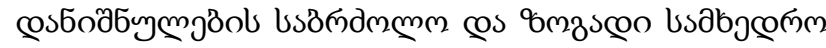

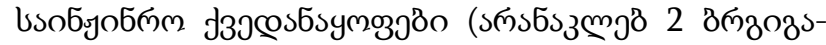

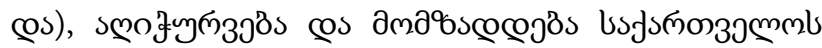

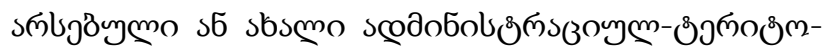

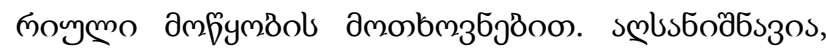

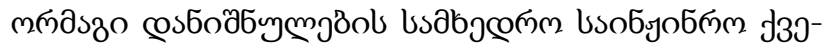

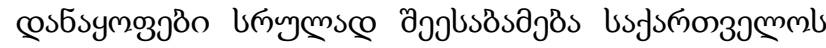

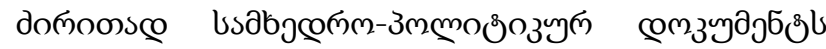

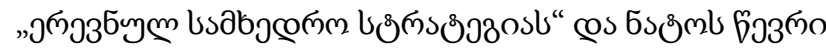

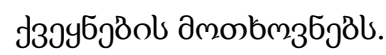

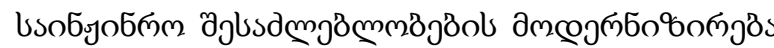

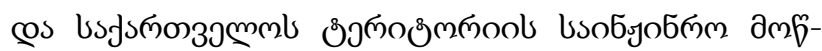
ym

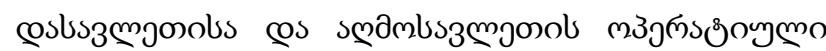

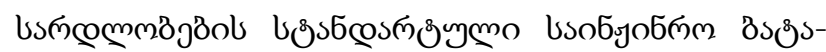

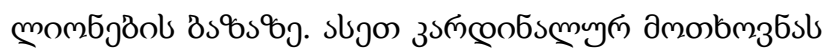

ISSN 1512-0996

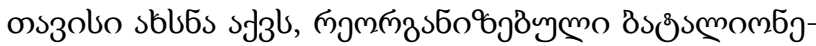

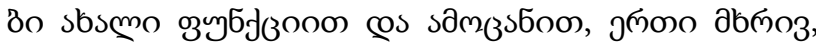

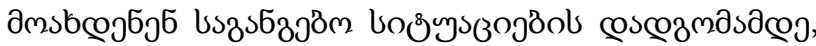

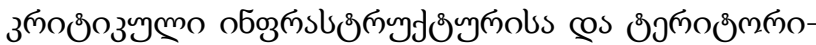

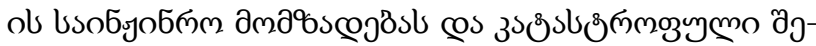

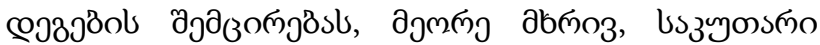

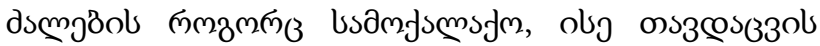

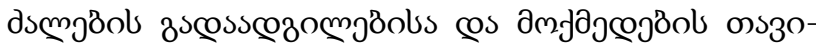

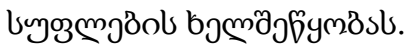

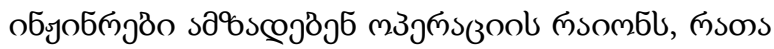

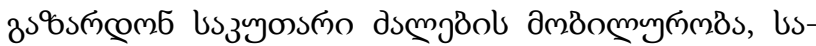

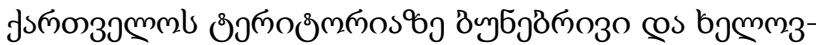

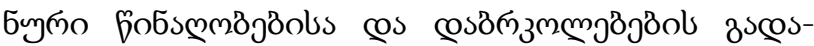

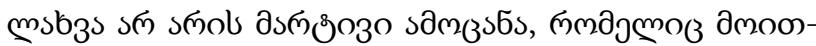

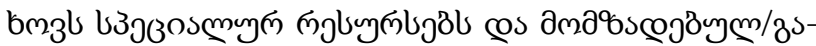

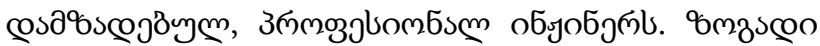

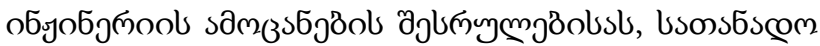

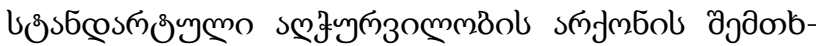

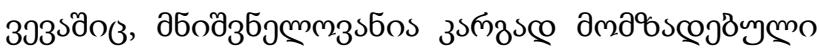

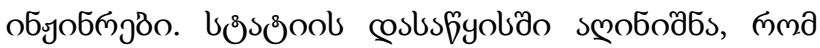

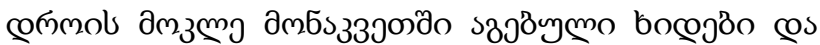

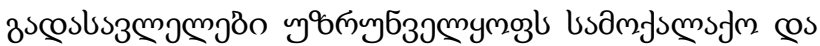

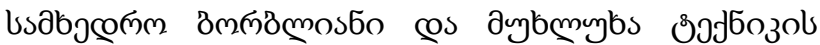

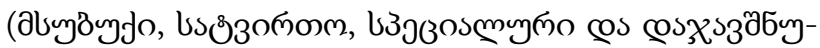

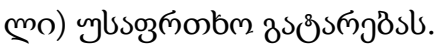

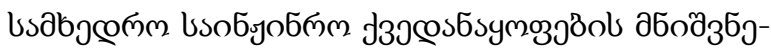

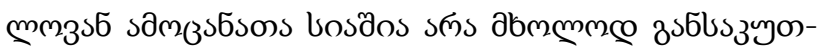

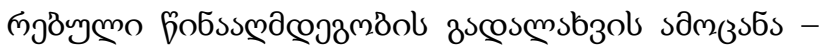

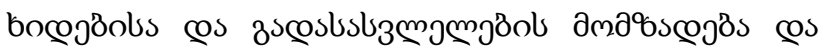

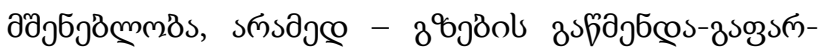

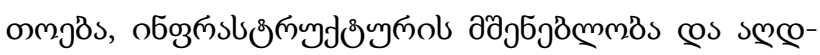

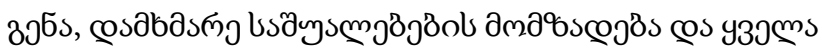

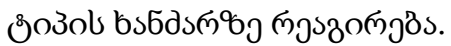




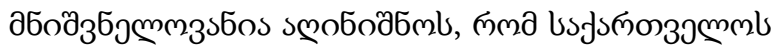

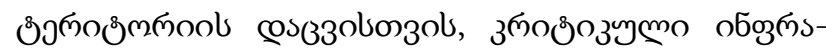

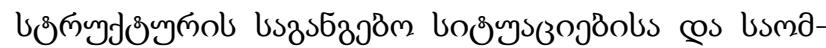

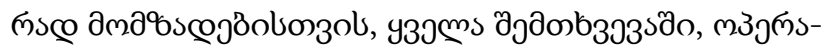

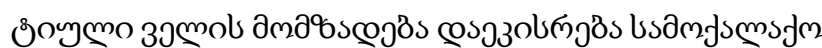

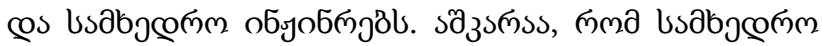

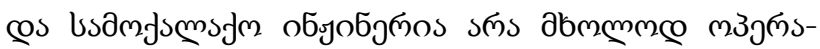

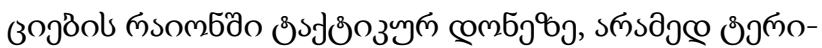

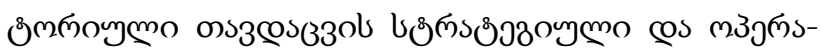

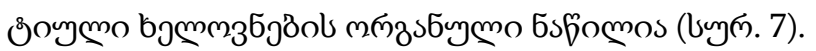

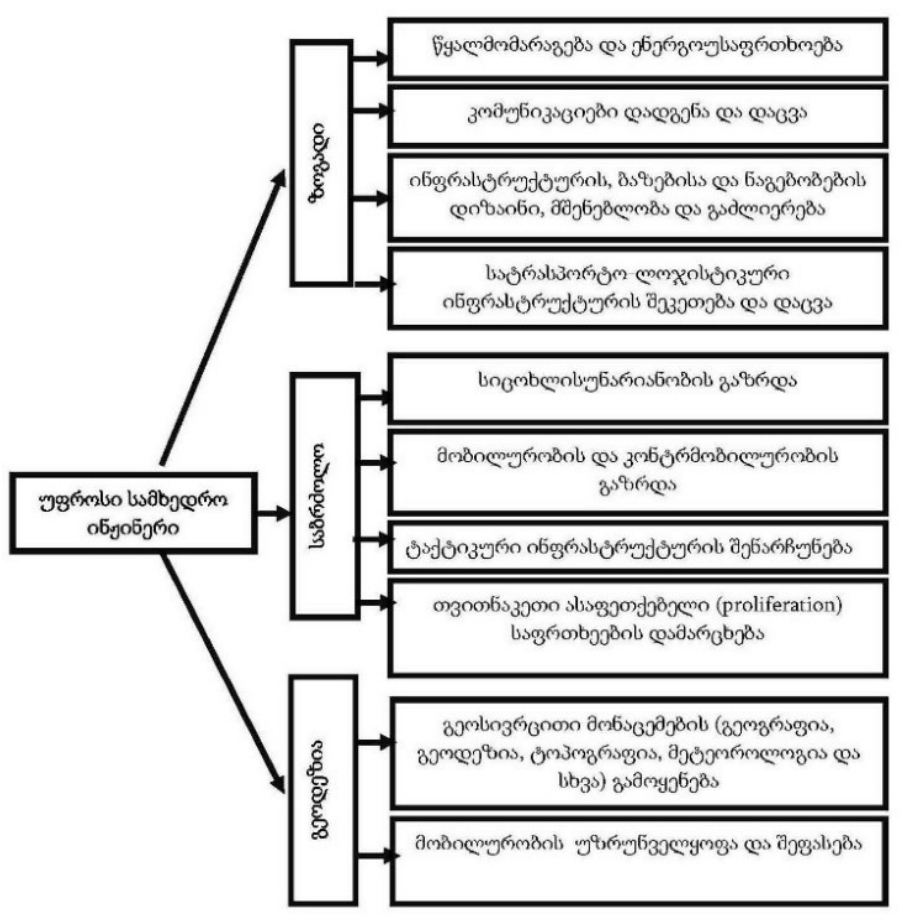

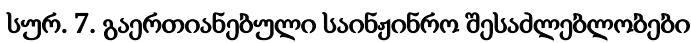

\section{cost $336 s$}

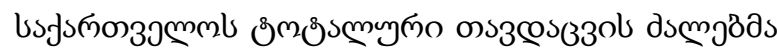

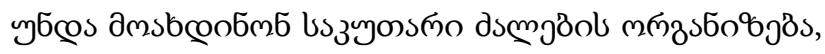

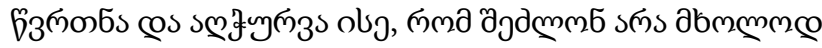

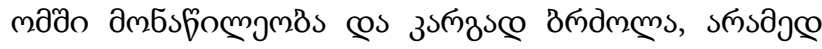

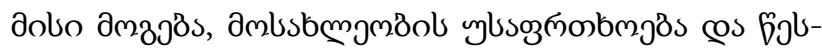

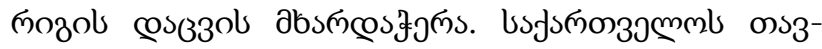

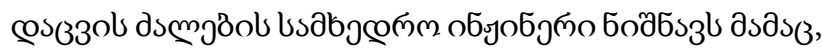

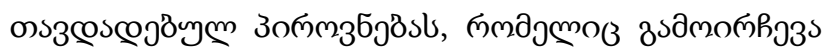

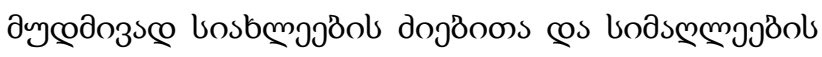

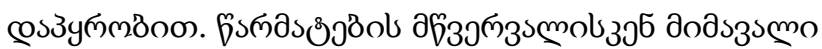

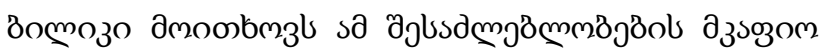

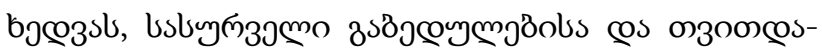

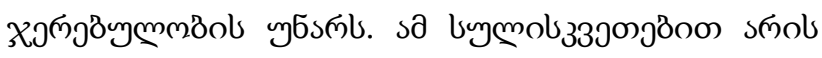

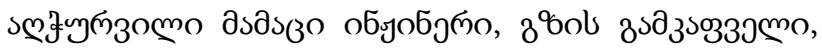

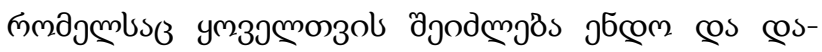

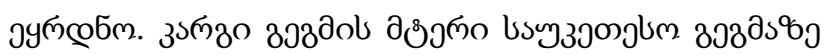

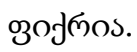




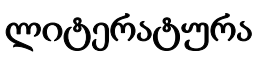

1. Medzmariashvili E. Fundamentals of Georgian military engineering doctrine. Tbilisi. 2006. (in Georgian).

2. Military encyclopedic dictionary of Georgia. Tbilisi. 2017. (In Georgian).

3. URL: http://pprdeast2.eu/wp-content/uploads/2015/08/GE-Country-Profile-GE.pdf (in Georgian).

UDC 355/359

SCOPUS CODE 2205

\title{
Restoration of civil/military objects in the conditions of common and scarce engineering resources - Kodori Gorge
}

\author{
Murtaz Gujejiani Department of Civil and Industrial Construction, Georgian Technical University, 68 \\ M. Kostava str., 0160 Tbilisi, Georgia \\ E-mail: nav.qutaisi@gmail.com
}

\section{Reviewers:}

E. Medzmariashvili, Professor, Faculty of Civil Engineering, GTU

E-mail: e.medzmariashvili@gtu.ge

T. Shubladze, Chief Scientific Researcher, Institute of Constructions, Special Systems and Engineering Provision, GTU

E-mail: shubladze.tengiz@gmail.com

Abstract. The present article provides a theoretical and practical example of the effective management and use of Georgian civil and military engineering resources. The state's defense system is complex. Unpredictable processes, conduction of modern wars, combat operations, battles and the nature of individual operations, the forms and content of their detection, territorial environment, and, the most importantly, the threats posed by strict and necessary requirements stipulate the joint and combined operation of civilian and military components, their clearly defined roles, functions and tasks.

The examples and the structure of participatory design are given, which fully correspond to the capabilities of creating an artificial environment in accordance with the requirements of total defense. In case of total defense - in emergency and military situations - they play an important role, largely related to critical infrastructure and engineering preparations for the defense of the territory of Georgia.

Key words: Military engineer; preparation of operations area; synthesis of the country's engineering resources; total defense. 


\title{
UDC 355/359
}

SCOPUS CODE 2205

\section{В условиях единого и дефицитного инженерного потенциала, восстановления объектов общего гражданско-военного значения - Кодорского ущелья}

\author{
Муртаз Гуджеджиани Департамент гражданского и промышленного строительства им. Агули Сохадзе, \\ Грузинский технический университет, Грузия, 0160, Тбилиси, ул. М. Костава, $68^{\mathrm{a}}$ \\ E-mail: nav.qutaisi@gmail.com
}

\section{Рецензенты:}

Э. Медзмариашвили, профессор строительного факультета ГТУ

E-mail: e.medzmariashvili@gtu.ge

Т. Шубладзе, главный научный сотрудник Института специальных систем сооружений и инженерного обеспечения ГТУ

E-mail: shubladze.tengiz@gmail.com

Аннотация. В данной статье представлен теоретический и практический пример эффективного управления и использования грузинских гражданских и военных инженерных ресурсов. Система обороны государства сложна. Непредсказуемые процессы, ведение современных войн, боевых действий, сражений и характер отдельных операций, формы и содержание их обнаружения, территориальная среда и, что самое важное, угрозы, создаваемые строгими и необходимыми требованиями, составляют совместную и совмещенную операцию гражданского и военного компонентов. Четко определенные роли, функции и задачи.

В статье «В условиях единого и дефицитного инженерного потенциала, восстановления объектов общего гражданско-военного значения - Кодорского ущелья» приведены примеры и структура совместного проектирования, полностью соответствующие возможностям создания искусственной среды в соответствии с требованиями тотальной обороны. И в случае тотальной обороны - в чрезвычайных и военных ситуацияхиграют важную роль, в значительной степени связанные с критической инфраструктурой и инженерными приготовлениями для защиты территории Грузии.

Ключевые слова: военный инженер; подготовка района боевых действий; синтез инженерных ресурсов страны; тотальная оборона.

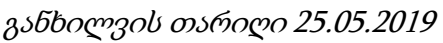

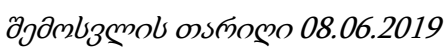

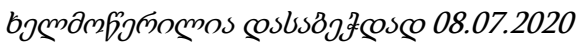

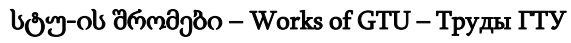

ISSN 1512-0996 